$01 ; 14$

\title{
Использование вейвлетов для распознавания типа движения по данным электрической активности головного мозга
}

\author{
() Д.С. Гришина ${ }^{1}$, А.Н. Павлов ${ }^{1,2, \uparrow, ~ О . Н . ~ П а в л о в а ~}{ }^{2}$, А.Е. Руннова ${ }^{3}$ \\ ${ }^{1}$ Саратовский государственный технический университет им. Гагарина Ю.А., Саратов, Россия \\ ${ }^{2}$ Саратовский национальный исследовательский государственный университет им. Н.Г. Чернышевского, Саратов, Россия \\ ${ }^{3}$ Университет Иннополис, Иннополис, Россия \\ E-mail: pavlov.alexeyn@gmail.com
}

Поступило в Редакцию 29 апреля 2019 г.

В окончательной редакции 29 апреля 2019 г.

Принято к публикации 8 мая 2019 г.

Рассматривается задача распознавания осцилляторных паттернов электроэнцефалограмм при осуществлении движений и их мысленного представления для реализации программной части нейроинтерфейса. С использованием многомасштабного анализа проведена оценка числа каналов, обеспечивающих достоверное разделение фоновой активности и движений различного типа.

Ключевые слова: сигнал, распознавание паттернов, вейвлет-анализ, фильтрация.

DOI: $10.21883 / 0000000000$

Достигнутые успехи в создании интерфейсов мозгкомпьютер (ИМК) [1-5] привели к формированию новой области междисциплинарных исследований, в которой за последние годы был предложен ряд оригинальных разработок. ИМК осуществляют распознавание характерных особенностей регистрируемых сигналов, например электрических сигналов головного мозга - электроэнцефалограмм (ЭЭГ), и последующее формирование команд управления аппаратной частью. Они позволяют совершать различные действия в окружающей среде на основе мысленных намерений, без использования мышц [6-10], что является крайне важным для людей с нарушениями двигательных функций. Существующие в настоящее время ИМК обеспечивают возможность парализованным людям управлять движением курсора на экране монитора, синтезировать голосовые сообщения, регулировать движения и т.д. Ключевую роль при создании ИМК выполняет программное обеспечение для детектирования и распознавания паттернов, соответствующих разным мысленным намерениям. Оно должно осуществлять быстрое выявление характерных признаков паттернов по коротким фрагментам сигналов с учетом их вариабельности, что является сложной задачей и требует привлечения специальных методов [10-15]. С этой целью могут применяться, например, подходы на основе вейвлет-анализа $[16,17]$ или флуктуационного анализа $[18,19]$. Такой инструментарий позволяет решать задачи распознавания движений, но при этом методы, использованные в [17], не обеспечивают высокое быстродействие. С точки зрения скорости обработки данных целесообразно выбирать быстрые алгоритмы на основе дискретного вейвлет-преобразования и соответствующих базисных функций. В настоящей работе рассматриваются возможности и ограничения этого подхода применительно к решению задачи распознавания типа движений нетренированных людей.

Эксперименты проводились на девяти здоровых добровольцах. Сигналы ЭЭГ регистрировались электроэнцефалографом „Энцефалан“. Помимо стандартной установки 10-20 применялись промежуточные электроды, позволяющие увеличить число каналов до 32 (схема 10-10). Распознавание паттернов проводилось по предварительно записанным сигналам ЭЭГ для сопоставления возможностей и ограничений анализа на основе разных базисов. В ходе экспериментов записывались фоновая активность $(10 \mathrm{~min})$ и трехсекундные фрагменты ЭЭГ при выполнении движений (подъем правой/левой руки или правой/левой ноги). Дополнительно испытуемых просили мысленно представить эти движения и записывали сигналы, соответствующие воображению моторных функций. Каждый тип реальных и воображаемых движений регистрировался не менее 40 раз для обеспечения достаточной статистики, при этом выбирался случайный порядок движений, чтобы снизить влияние адаптации при выполнении повторяющихся действий.

Обработка экспериментальных данных проводилась на основе многомасштабного анализа [20], который осуществляет разложение сигнала с использованием набора зеркальных фильтров: низкочастотных $\varphi_{j, k}(t)$ и высокочастотных $\psi_{j, k}(t)$, формируемых путем масштабных преобразований и смещений соответственно, скейлингфункции $\varphi(t)$ и вейвлета $\psi(t)$

$$
\varphi_{j, k}=2^{j / 2} \varphi\left(2^{j} t-k\right), \quad \psi_{j, k}=2^{j / 2} \psi\left(2^{j} t-k\right) .
$$

В качестве базисных функций были выбраны вейвлеты семейства Добеши [20]. Разложение сигнала на некотором уровне разрешения $m$ осуществлялось по быстрой 


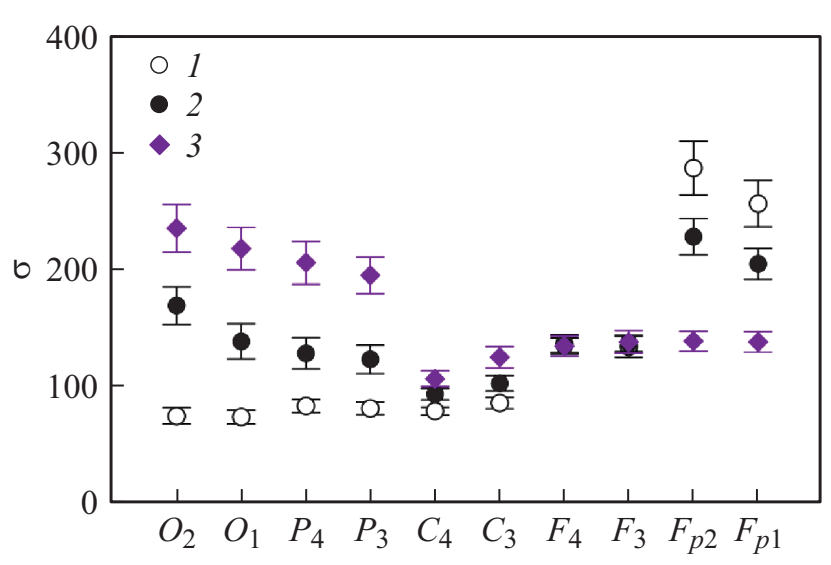

Рис. 1. Зависимость значения $\sigma$ от выбора канала регистрации ЭЭГ. Обозначение каналов приведено в соответствии с международной схемой расстановки 10-10. Порядок расположения электродов соответствует смещению от затылочной области ко лбу. Цифрами показаны результаты расчета для фоновой активности (1), реальных (2) и воображаемых (3) движений.

(пирамидальной) схеме следующим образом:

$$
x(t)=\sum_{k} s_{m, k} \varphi_{m, k}(t)+\sum_{j \geqslant m} \sum_{k} d_{j, k} \psi_{j, k}(t) .
$$

Количественным критерием, характеризующим вариабельность коэффициентов $d_{j, k}$, являлась дисперсия

$$
\sigma(j)=\sqrt{\frac{1}{M} \sum_{k=0}^{M-1}\left[d_{j, k}-\left\langle d_{j, k}\right\rangle\right]^{2}}
$$

В зависимости от масштаба $j$ число коэффициентов разложения $M$ меняется. Как отмечается в [21], диагностика режимов динамики во многих прикладных задачах обеспечивается выбором подходящего значения $j$, например 4 или 5. С учетом этих исследований, а также результатов нашего предварительного анализа сигналов ЭЭГ далее приводятся результаты для меры $\sigma=\sigma(5)$.

Сначала решалась сравнительно простая задача — отличить реальные и воображаемые движения от фоновой активности. Для этого был выбран один тип движений (подъем левой руки), результаты расчета $\sigma$ были сопоставлены для разных расположений электродов. Как видно из рис. 1, выбор электрода оказывает существенное влияние. Например, в затылочной области (электроды $O_{1}$ и $O_{2}$ ) дисперсия вейвлет-коэффициентов при реальных и, особенно, при воображаемых движениях значительно превышает величину $\sigma$, соответствующую фоновой активности, тогда как для электродов, расположенных в области лба $\left(F_{p 1}\right.$ и $\left.F_{p 2}\right)$, наблюдается противоположный эффект. Отметим, что выбор электродов, обеспечивающих наиболее сильные различия паттернов ЭЭГ, зависит от испытуемого. Качество распознавания можно также улучшить за счет выбора подходящего вейвлет-базиса. В проводимых исследованиях было осу- ществлено сравнение различных базисных функций, различия между паттернами ЭЭГ оценивались по критерию Стьюдента. В большинстве экспериментов наилучшие результаты были получены для вейвлета $D^{8}$, который был выбран для более детальных сопоставлений различных паттернов.

Далее анализировалась возможность разделения типов движений и проводилось сравнение участков ЭЭГ, соответствующих движениям рук и ног. В соответствии с данными рис. 2, $a$ они диагностируются с сопоставимой точностью: достоверные различия наблюдаются в 21-22 каналах из 32. Воображаемые движения также надежно различаются, хотя и в меньшем числе каналов (16-17 из 32). Более сложной задачей является поиск различий между типами движений - левой и правой руки или ноги. Как видно из рис. $2, b$, такие различия достоверно диагностируются только в 6-7 каналах из 32 для реальных движений и лишь в 1-2 каналах для воображаемых
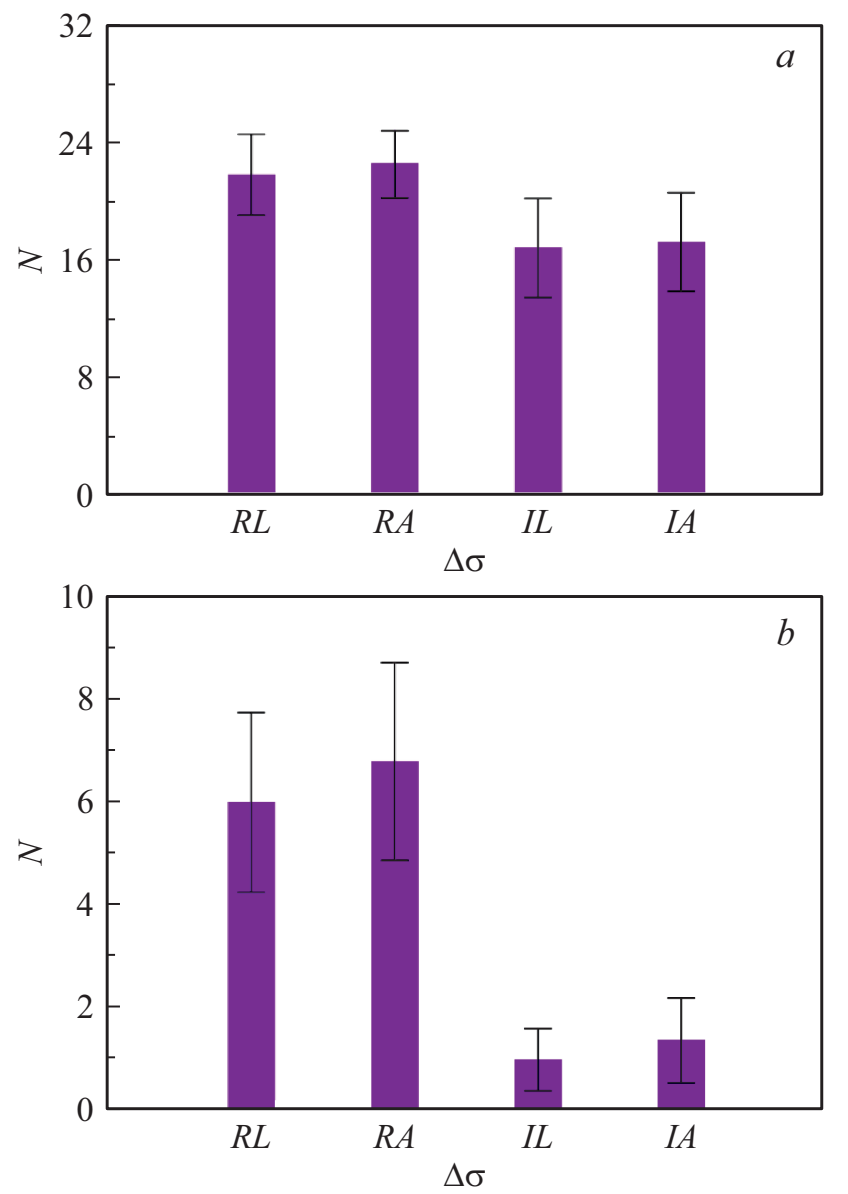

Рис. 2. Число каналов, по которым проводится достоверное $(p<0.01)$ распознавание выбранного типа движения от фоновой активности $(a)$ и от других типов движений $(b)$. Результаты представлены для движений ноги $(R L)$ и руки $(R A)$, а также для воображаемых движений ноги $(I L)$ и руки $(I A)$. Расчеты для движений правой и левой ноги/руки проводились по отдельности; с учетом того, что результаты были похожими, на рисунке приводятся усредненные данные, представленные в виде средних значений и стандартной ошибки. 
движений. При этом следует отметить, что у некоторых испытуемых число каналов намного выше, чем у других (одним из ключевых факторов является опыт испытуемых в мысленном представлении движений). В частности, для испытуемых, которые повторно участвовали в исследованиях, увеличивалось число каналов, позволяющих распознавать мысленные намерения. Таким образом, аппарат многомасштабного анализа может использоваться в интерфейсах мозг-компьютер, однако при этом необходимы предварительная тренировка и выбор каналов с учетом индивидуальных особенностей испытуемых.

\section{Финансирование работы}

Исследование выполнено при поддержке гранта Российского научного фонда (проект № 17-72-30003).

\section{Соблюдение этических стандартов}

Все процедуры, выполненные в исследовании с участием людей, соответствуют этическим стандартам институционального и национального комитета по исследовательской этике и Хельсинкской декларации 1964 г. и ее последующим изменениям или сопоставимым нормам этики. От каждого из включенных в исследование участников было получено информированное добровольное согласие.

\section{Конфликт интересов}

Авторы заявляют, что у них нет конфликта интересов.

\section{Список литературы}

[1] Birbaumer N., Ghanayim N., Hinterberger T., Iversen I., Kotchoubey B., Kübler A., Perelmouter J., Taub E., Flor H. // Nature. 1999. V. 398. P. 297-298.

[2] O'Doherty J.E., Lebedev M.A., Ifft P.J., Zhuang K.Z., Shokur S., Bleuler H., Nicolelis M.A. // Nature. 2011. V. 479. P. 228-231.

[3] Wolpaw J.R., McFarland D.J. // PNAS. 2004. V. 101. P. 17849-17854.

[4] Brain-computer interfaces: principles and practice / Eds J.R. Wolpaw, E.W. Wolpaw. N.Y.: Oxford University Press, 2012. 419 p.

[5] Chen X., Wang Y., Nakanishi M., Gao X., Jung T.-P., Gao S. // PNAS. 2015. V. 112. P. E6058-E6067.

[6] Bowsher K., Civillico E.F., Coburn J., Collinger J., ContrerasVidal J.L., Denison T., Donoghue J., French J., Getzoff N., Hochberg L.R., Hoffmann M., Judy J., Kleitman N., Knaack G., Krauthamer V., Ludwig K., Moynahan M., Pancrazio J.J., Peckham P.H., Pena C., Pinto V., Ryan T., Saha D., Scharen H., Shermer S., Skodacek K., Takmakov P., Tyler D., Vasudevan S., Wachrathit K., Weber D., Welle C.G., Ye M. // J. Neural Eng. 2016. V. 13. P. 023001.

[7] Ma T., Li H., Deng L., Hao Y., Xulin L., Peiyang L., Fali L., Rui Z., Tiejun L., Dezhong Y., Peng X. // J. Neural Eng. 2017. V. 14. P. 026015.
[8] Peternel L., Noda T., Petrič T., Ude A., Morimoto J., Babič J. // PLoS One. 2016. V. 11. P. e0148942.

[9] Maksimenko V.A., van Heukelum S., Makarov V.V., Kelderhuis J., Lüttjohann A., Koronovskii A.A., Hramov A.E., van Luijtelaar G. // Sci. Rep. 2017. V. 7. P. 2487.

[10] Choi I., Rhiu I., Lee Y., Yun M.H., Nam C.S. // PLoS One. 2017. V. 12. P. e 0176674.

[11] Божсокин С.В. // ЖТФ. 2010. Т. 80. В. 9. С. 16-24.

[12] Грубов В.В., Руннова А.Е., Короновский А.А., Храмов А.Е. // Письма в ЖТФ. 2017. Т. 43. В. 13. С. 58-64.

[13] Колоскова А.Д., Москаленко О.И. // Письма в ЖТФ. 2017. T. 43. B. 10. C. $102-110$.

[14] Грубов В.В., Недайвозов В.О. // Письма в ЖТФ. 2018. T. 44. B. 10. C. 103-110.

[15] Фролов Н.С., Писарчик А.Н. // Письма в ЖТФ. 2018. Т. 44. B. 10 . C. $76-85$.

[16] Короновский А.А., Макаров В.А., Павлов А.Н., Ситникова Е.Ю., Храмов А.Е. Вейвлеты в нейродинамике и нейрофизиологии. М.: Физматлит, 2013. 271 с.

[17] Maksimenko V.A., Pavlov A., Runnova A.E., Nedaivozov V., Grubov V., Koronovskii A., Pchelintseva S.V., Pitsik E., Pisarchik A.N., Hramov A.E. // Nonlinear Dynamics. 2018. V. 91. P. 2803-2817.

[18] Pavlov A.N., Runnova A.E., Maksimenko V.A., Pavlova O.N., Grishina D.S., Hramov A.E. // Physica A. 2018. V. 509. P. 777-782.

[19] Павлов А.Н., Руннова А.Е., Максименко В.А., Павлова О.Н., Гришина Д.С., Храмов А.Е. // Письма в ЖТФ. 2019. T. 45. B. 4. C. $8-10$.

[20] Daubechies I. Ten lectures on wavelets. Philadelphia: S.I.A.M., 1992. $378 \mathrm{p}$.

[21] Дремин И.М., Иванов О.В., Нечитайло В.А. // УФН. 2001. T. 171. № 5. C. 465-501. 